\title{
Formulation of Huangqin Decoction influence immune function and fecal microbiome of chicks suffered Escherichia coli 078 challenge
}

\author{
Xichun Zhang ( $\nabla$ zxcxpy@126.com ) \\ Shanxi University of Chinese Medicine,Yuci
}

Shuming Chen

Shanxi Agricultural University, Taigu

Junyan Wang

Shanxi Agricultural University, Taigu

Rui Li

Shanxi University of Chinese Medicine,Yuci

Yong Liu

Shanxi University of Chinese Medicine,Yuci

Chensheng Gu

Shanxi University of Chinese Medicine,Yuci

Haili Wang

Shanxi University of Chinese Medicine,Yuci

Jianjian Feng

Shanxi Agricultural University, Taigu

Linjie Bao

Shanxi Agricultural University, Taigu

Yihe Wu

Shanxi Agricultural University, Taigu

\section{Research Article}

Keywords: Huangqin Decoction, chick, immune function, fecal microbiome, Escherichia coli 078

Posted Date: January 28th, 2022

DOI: https://doi.org/10.21203/rs.3.rs-1126083/v2

License: (9) (1) This work is licensed under a Creative Commons Attribution 4.0 International License. Read Full License 


\section{Abstract}

Huangqin Decoction (HQD), a traditional Chinese medicine formula from the Shang Han Lun written by Zhang Zhongjing, has been used in China for nearly two thousand years. According to traditional Chinese medicine and a previous literature, HQD has the effect of clearing heat and removing toxin, antidiarrhea, and relieving pain. Therefore, HQD were used to prevent or cure many diseases, such as inflammation, diarrhea, malaria, and other acute or chronic gastrointestinal diseases.

HQD supplementation showed higher ADG and reduced the mortality rate caused by $E$. coli 078 challenge, decreased the heart index and liver index, and increased the bursal index and spleen index. HQD supplementation decreased the serum LZM, IL-1 $\beta$, TNF-a, IL-10, IL-6 levels, down-regulated the mRNA expression of TLR4, TLR5 and TLR15 in the spleen in E. coli O78 challenged chicks, and up-regulated the mRNA expression of TLR4, TLR5 and TLR15 in the bursal in that. At the phylum levels, HQD supplementation reversed the increased of OTUs, decreased the relative abundance of harmful bacteria Proteobacteria, increased the relative abundance of probiotics Bacteroidetes and Firmicutes. At the genus levels, HQD decreased the relative abundance of harmful bacteria Escherichia-Shigella and Pseudomonas. It means that HQD reversed the change of the gut microbiota structure. Compared with HQD, HQD-DZ and HQD-HQ increased the mortality rate. HQD-HQ decreased the ADG and liver index. HQD-GC decreased the spleen index. HQD-DZ, HQD-HQ, HQD-SY and HQD-GC increased the serum TL-6, but only the HQD-HQ and HQD-SY increased the serum TNF-a. All herb absent HQD did not activate the toll-like receptors signaling pathways in spleen and bursal of chicks. HQD-DZ and HQD-HQ increased the harmful bacteria Escherichia-Shigella, and HQD-DZ increased the harmful bacteria Proteobacteria levels.

In a word, dietary supplementation with HQD, by down-regulating the mRNA expression of TLR4, TLR5 and TLR1 5 in the spleen, further decreasing the serum LZM and IL-1ß, TNF-a, IL-10, IL-6 levels, improves the immune function and reverse the change of fecal microbiome in chicks challenged with $E$. coli O78. About herb absent groups, the results shown that SY and DZ play a key role in reducing the level of inflammatory factors and keeping fecal microbiome balance respectively. what's more, we highlighted that $\mathrm{HQ}$ is indispensable in $H Q D, H Q$ not only play a key role in reducing the level of inflammatory factors, but also keep the balance of fecal microflora.

\section{Introduction}

Colibacillosis refers to any localized or systemic infection caused by avian pathogenic Escherichia coli, caused by specific serotypes or opportunistically pathogenic Escherichia coli, is one of the crucial bacterial diseases of poultry ${ }^{1}$. Young birds, in which the protective immune system is not fully developed, are more vulnerable. E. coli serotypes 01,02 , and 078 are the most found in domestic breeds with colibacillosis ${ }^{2}$. Although various antibiotics are typically used to prevent and control colibacillosis, cumulative reports have demonstrated that drug resistance of E.coli 078 has increased owing to the spreading of resistance genes such as extended spectrum beta-lactamases (ESBL) and/or plasmid-mediated Amp-C beta-lactamases (Amp-C) ${ }^{3}$. Therefore, potential antibiotic alternatives to reduce antimicrobial drug usage in poultry production are urgently needed. 
HQD, a traditional Chinese medicine formula from the Shang Han Lun written by Zhang Zhongjing, has been used in China for nearly two thousand years ${ }^{4}$. The classical formula consists of four components: Scutellariae Radix, Paeoniae Radix Alba, Jujubae Fructus, Licorice. Clinical studies have shown that HQD is safe and effective for treatment of complex gastrointestinal symptoms, like ulcerative colitis and associated cancer. The researchers suggest that this is because HQD could regulate the structure of intestinal flora and inhibit the intestinal inflammatory signaling pathway ${ }^{5}$. Basic studies have shown that the main effectors of HQD are baicalin, paeoniflorin, polysaccharides and flavonoids, which can regulate the number of mast cells and downregulate the expression of inflammatory factors through the activation of TLR4/MyD88/NF-KB, IL6/JAK/STAT3 and STAT3/NF-KB/IL-6 signaling pathways ${ }^{6}$, thus play immune regulation and antiinflammatory effect ${ }^{7}$. HQD had no inhibitory effect on Escherichia coli 078 in vitro, but the antibacterial effect was enhanced after intestinal flora metabolism ${ }^{8}$, indicating that the bacteriostatic effect of HQD is mostly by regulating the metabolites and balance of intestinal bacteria rather than directly. Therefore, this study hypothesized that the HQD is effective for treatment of chicken colibacillosis by regulating the immune function and intestinal microflora structure.

To investigate the molecular mechanisms of HQD in chick model of artificially infected chicken colibacillosis, the expression levels of lysozyme, inflammatory cytokines in serum, and Toll-like receptors in spleen and bursal were measured. Furthermore, the gut microbiota was profiled using Illumina HiSeq 2500 sequencing of the bacterial 16S rDNA gene V3-V4 region.

\section{Results}

\section{1. $H Q D$ reduced the mortality of colibacillosis and increased the average daily gain in chicks}

Compared with the CG, chicks challenged with E. coli 078 at 27 days of age showed ruffled feathers, closed eyes, reluctance to move, defecated white or green stools and soiled vent. Post-mortem examination showed that $E$. coli 078 infection led to severe perihepatitis, pericarditis, granuloma, and bleeding spots in the small intestine (Fig. 1). E. coli 078 infection reduced average daily gain (ADG) between 1 and 32 days of age $(p<0.05)$, which was reversed by HQD (Fig. 2A). There was no significant difference between HQD-HQ, HQD-DZ, HQD-GC, HQD-SY and ENR supplementation compared with HQD. E. coli O78 infection increased mortality at day 5 post E. coli 078 challenge, which was reversed by HQD, 500 mg/kg HQD-GC and 500 mg/kg HQD-SY (Fig. 2B). Moreover, there was no effect on mortality in chicks challenged with E. coli 078 at the dose of 250 $\mathrm{mg} / \mathrm{kg}$ except for the HQD group. Therefore, the dose of $500 \mathrm{mg} / \mathrm{kg}$ groups were more worthy of further study.

\subsection{HQD changed the visceral index in chicks with colibacillosis}

In contrast, the heart index and the liver index were increased in chicks challenged with E. coli O78, as compared with control group, which was reversed by HQD and ENR. Chicks with supplementation challenged with E. coli 078 led to reduced visceral index in the bursal, which was reversed by HQD and ENR. Chicks 
challenged with E. coli 078 were no effect on spleen index, which was increased by HQD. Compared with HQD, HQD-DZ and HQD-SY have the same effect as HQD, HQD-GC resulted in decreased spleen index, while HQD-HQ resulted in increased liver index in chicks challenged with E. coli O78. The results showed that HQD exerted significant protective effects against the organ index of artificial induced colibacillosis, in which HQ played the main role (Table 1$)$.

\section{Table 1}

Visceral index of chicks in Control group (CG), Model group (MG), Huangqin Decoction group (HQD); and that in HQD group of DZ absent (HQD-DZ), GC absent (HQD-GC), SY absent (HQD-SY), HQ absent (HQD-HQ); and in Enrofloxacin group (ENR). The different letter superscript in the same column indicates that the difference is significant ( $p \varangle 0.05)$.

\begin{tabular}{|lllll|}
\hline & Spleen & Bursal & Heart & Liver \\
\hline CG & $1.89 \pm 0.44^{\mathrm{b}}$ & $5.84 \pm 0.25^{\mathrm{a}}$ & $6.91 \pm 0.54^{\mathrm{c}}$ & $26.20 \pm 0.40^{\mathrm{c}}$ \\
\hline MG & $2.68 \pm 0.84^{\mathrm{b}}$ & $2.77 \pm 1.21^{\mathrm{b}}$ & $15.05 \pm 1.64^{\mathrm{a}}$ & $72.43 \pm 16.63^{\mathrm{a}}$ \\
\hline HQD & $3.79 \pm 0.37^{\mathrm{a}}$ & $4.67 \pm 2.12^{\mathrm{ab}}$ & $7.83 \pm 1.43^{\mathrm{bc}}$ & $37.71 \pm 9.08^{\mathrm{c}}$ \\
\hline HQD-DZ & $3.04 \pm 0.44^{\mathrm{ab}}$ & $3.14 \pm 0.88^{\mathrm{b}}$ & $7.14 \pm 0.89^{\mathrm{c}}$ & $34.41 \pm 7.05^{\mathrm{c}}$ \\
\hline HQD-GC & $2.55 \pm 0.91^{\mathrm{b}}$ & $2.13 \pm 1.06^{\mathrm{b}}$ & $8.12 \pm 1.37^{\mathrm{bc}}$ & $42.55 \pm 3.00^{\mathrm{bc}}$ \\
\hline HQD-SY & $3.31 \pm 0.90^{\mathrm{ab}}$ & $3.28 \pm 1.22^{\mathrm{b}}$ & $7.90 \pm 0.61^{\mathrm{bc}}$ & $39.25 \pm 2.38^{\mathrm{bc}}$ \\
\hline HQD-HQ & $2.81 \pm 0.68^{\mathrm{ab}}$ & $3.16 \pm 1.23^{\mathrm{b}}$ & $9.27 \pm 0.64^{\mathrm{b}}$ & $49.78 \pm 4.12^{\mathrm{b}}$ \\
\hline ENR & $3.29 \pm 0.83^{\mathrm{ab}}$ & $5.36 \pm 1.92^{\mathrm{a}}$ & $8.02 \pm 1.12^{\mathrm{bc}}$ & $34.71 \pm 5.44^{\mathrm{c}}$ \\
\hline
\end{tabular}

\subsection{Effect of HQD on E. coli O78-induced lysozyme in serum}

E. coli 078 infection increased the protein levels of lysozyme in serum, as compared with that of control group, which was significantly reversed by HQD and ENR administration. HQD-GC and HQD-SY have the same effect as HQD, but HQD-DZ and HQD-HQ couldn't reverse the increase in lysozyme protein level. The results showed that $\mathrm{HQ}$ and $\mathrm{DZ}$ in $\mathrm{HQD}$ played an important role in eliminating the effect of $E$. coli 078 on lysozyme (Fig. 3).

\subsection{Effect of HQD on E. coli O78-induced inflammatory cytokines in serum}

ELISA assay was conducted to determine protein level of inflammatory cytokines in serum. E. coli 078 infection increased the protein levels of IL-1 $\beta$, TNF- $\alpha$ and IL-10 in serum, as compared with control group, indicating activation of inflammatory response following $E$. coli 078 infection. These alterations were 
significantly reversed by HQD and ENR administration. The protein level of IL-6 was increased by E. coli 078 challenged in serum of chicks, as compared with control group, however, HQD reduced its protein levels to even below control level. The results showed that HQD could reduce the inflammatory reaction in chicks challenged by $E$. coli 078 for 5 days. Compared with the HQD group, the protein levels of IL-1 $1 \beta$ and IL-10 were not different, while the levels of IL-6 protein were significantly increased in all herbs absent HQD. And the level of TNF-a protein in HQD-SY and HQD-HQ groups were significantly higher. The results indicated that the herbs in HQD have the effect of down-regulating pro-inflammatory factors IL-6. Meanwhile, HQ and SY play a key role in down-regulating TNF-a (Table 2).

\section{Table 2}

The level of inflammatory factor in serum of chicks in Control group (CG), Model group (MG), Huangqin Decoction group (HQD); and that in HQD group of DZ absent (HQD-DZ), GC absent (HQD-GC), SY absent (HQDSY), HQ absent (HQD-HQ); and in Enrofloxacin group (ENR). The different letter superscript in the same column indicates that the difference is significant ( $p \otimes 0.05)$.

\begin{tabular}{|lllll|}
\hline & IL-1 $\beta$ & IL-6 & TNF-a & IL-10 \\
\hline CG & $48.65 \pm 8.61^{\mathrm{c}}$ & $30.82 \pm 2.91^{\mathrm{c}}$ & $26.77 \pm 2.10^{\mathrm{d}}$ & $33.93 \pm 4.82^{\mathrm{bc}}$ \\
\hline MG & $106.71 \pm 5.38^{\mathrm{a}}$ & $70.72 \pm 2.39^{\mathrm{a}}$ & $72.82 \pm 3.58^{\mathrm{a}}$ & $66.96 \pm 2.39^{\mathrm{a}}$ \\
\hline HQD & $60.77 \pm 14.61^{\mathrm{bc}}$ & $20.11 \pm 1.60^{\mathrm{e}}$ & $24.38 \pm 5.93^{\mathrm{d}}$ & $37.95 \pm 7.91^{\mathrm{bc}}$ \\
\hline HQD-DZ & $47.67 \pm 7.22^{\mathrm{c}}$ & $38.29 \pm 2.40^{\mathrm{b}}$ & $30.39 \pm 3.17^{\mathrm{cd}}$ & $24.68 \pm 7.17^{\mathrm{c}}$ \\
\hline HQD-GC & $52.73 \pm 25.10^{\mathrm{c}}$ & $36.77 \pm 3.73^{\mathrm{b}}$ & $35.62 \pm 7.79^{\mathrm{cd}}$ & $30.90 \pm 13.98^{\mathrm{c}}$ \\
\hline HQD-SY & $70.92 \pm 29.76^{\mathrm{b}}$ & $24.58 \pm 2.86^{\mathrm{d}}$ & $49.89 \pm 11.95^{\mathrm{b}}$ & $43.69 \pm 28.43^{\mathrm{b}}$ \\
\hline HQD-HQ & $43.26 \pm 10.91^{\mathrm{c}}$ & $38.78 \pm 3.04^{\mathrm{b}}$ & $38.40 \pm 2.57^{\mathrm{c}}$ & $20.61 \pm 8.13^{\mathrm{c}}$ \\
\hline ENR & $61.75 \pm 20.39^{\mathrm{bc}}$ & $28.33 \pm 2.61^{\mathrm{c}}$ & $27.21 \pm 12.38^{\mathrm{d}}$ & $31.99 \pm 5.98^{\mathrm{bc}}$ \\
\hline
\end{tabular}

\subsection{Effect of HQD on mRNA level of Toll-like receptors in spleen and bursal of chicks}

Further to explore the effect of HQD on the immune function of chicks challenged with E. coli O78, the mRNA levels of TLR4, TLR5 and TLR15 in spleen and bursal were detected. Activation of Toll-like receptor signaling pathway plays an important role in defense against invading pathogens. E. coli 078 challenge enhanced mRNA level of TLR4, TLR5 and TLR15 in spleen, as compared with control group, indicating activation of the innate immune system in the spleen following $E$. coli 078 infection. And this alteration was significantly reversed by all administration. Compared with CG, E. coli 078 challenge had no effect on mRNA level of TLR4, TLR5 and TLR15 in bursal, but the mRNA levels were significantly increased after administration. The results 
indicated that the administration groups could regulate the anti-infection ability of chicks suffered E. coli 078 by increasing the mRNA levels of TLR4, TLR5 and TLR15 in the bursal. There was no significant difference between the results of other herb absent formulae and those of HQD group, indicating that HQD acted indirectly on immune organs (Table 3 ).

\section{Table 3}

The mRNA level of Toll-like receptor (TLR) in spleen and bursal of chicks in Control group (CG), Model group (MG), Huangqin Decoction group (HQD); and that in HQD group of DZ absent (HQD-DZ), GC absent (HQD-GC), SY absent (HQD-SY), HQ absent (HQD-HQ); and in Enrofloxacin group (ENR). The different letter superscript in the same column indicates that the difference is significant ( $p \bowtie 0.05$ ).

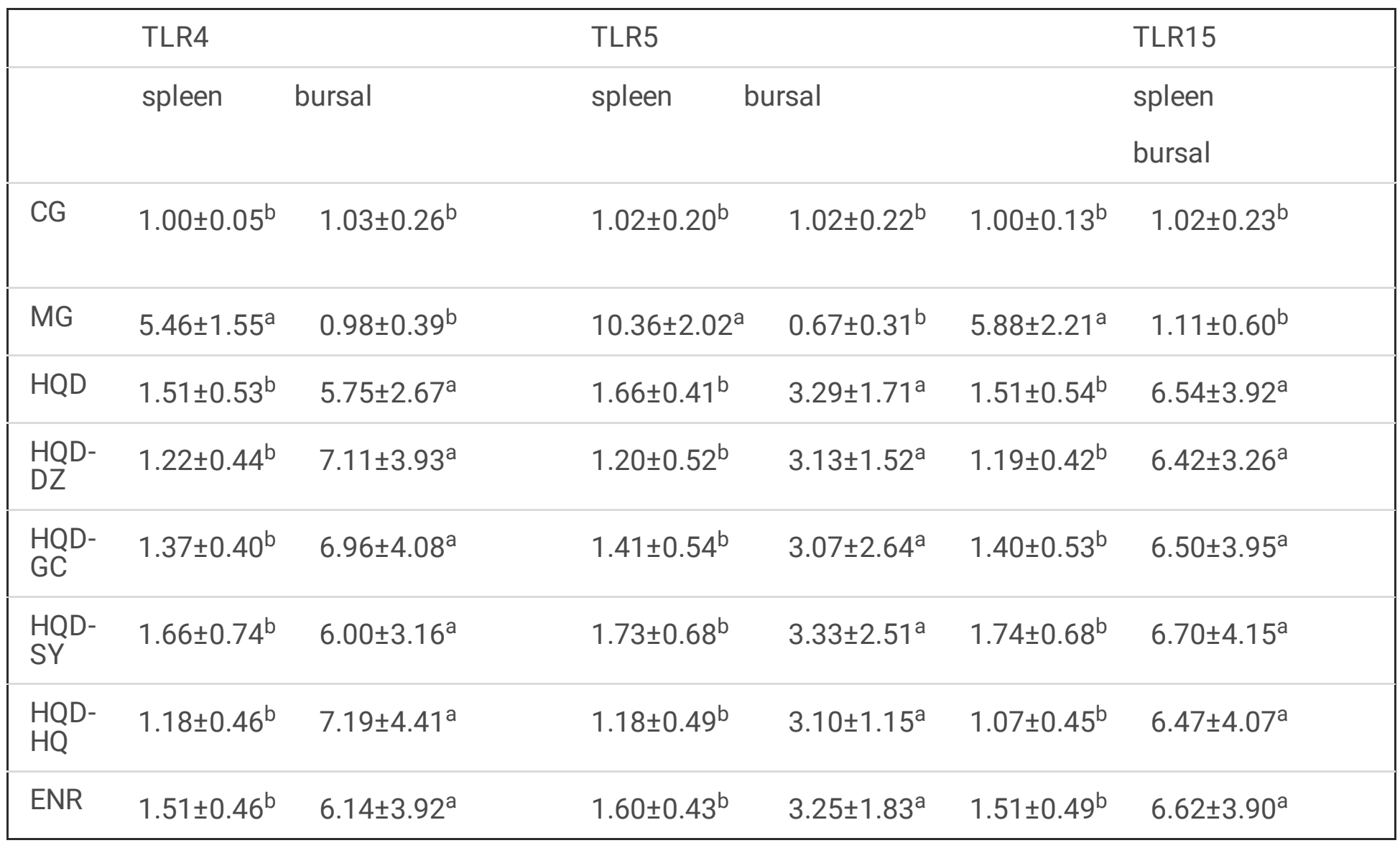

\subsection{The effect of HQD on gut microbiota in chick feces}

\subsubsection{HQD altered overall structural modulation of gut microbiota}

Structural changes in the gut microbiota have been reported in E. coli 078 models $^{9}$. Alpha diversity analysis showed a clear decline in bacterial diversity in response to $E$. coli 078 treatment, and a clear increase in diversity in response to HQD treatment. Compared with HQD group, the Shannon index of HQD-DZ group was 
significantly decreased, indicating that DZ had an important role in maintaining the intestinal microflora structure of chicks challenged with E. coli 078 (Table 4).

\section{Table 4}

The diversity of microbial in group of chicks in Control group (CG), Model group (MG), Huangqin Decoction group (HQD); and that in HQD group of DZ absent (HQD-DZ), GC absent (HQD-GC), SY absent (HQD-SY), HQ absent (HQD-HQ); and in Enrofloxacin group (ENR). The different letter superscript in the same column indicates that the difference is significant ( $p \bowtie 0.05)$.

\begin{tabular}{|lll|}
\hline & Chao1 & Shannon \\
\hline CG & $995.70 \pm 133.14^{\mathrm{a}}$ & $8.79 \pm 0.26^{\mathrm{a}}$ \\
\hline MG & $638.46 \pm 43.52^{\mathrm{b}}$ & $6.87 \pm 0.13^{\mathrm{b}}$ \\
\hline HQD & $884.87 \pm 119.90^{\mathrm{a}}$ & $8.55 \pm 1.26^{\mathrm{ab}}$ \\
\hline HQD-DZ & $518.60 \pm 42.76^{\mathrm{b}}$ & $2.78 \pm 0.77^{\mathrm{c}}$ \\
\hline HQD-GC & $823.83 \pm 268.85^{\mathrm{ab}}$ & $8.53 \pm 0.78^{\mathrm{ab}}$ \\
\hline HQD-SY & $455.77 \pm 127.88^{\mathrm{b}}$ & $6.76 \pm 1.06^{\mathrm{b}}$ \\
\hline HQD-HQ & $565.00 \pm 86.09^{\mathrm{b}}$ & $7.38 \pm 2.01^{\mathrm{ab}}$ \\
\hline ENR & $338.13 \pm 35.36^{\mathrm{b}}$ & $5.03 \pm 0.76^{\mathrm{b}}$ \\
\hline
\end{tabular}

A plateaued rarefaction curve of OTUs indicated that the sequencing depth covered all the species in the samples. E. coli 078 infection increased the OTUs, as compared with control group, which was reversed by $\mathrm{HQD}$ and ENR administration. Principal coordinate analysis (PCoA) showed similarity among samples, with similarity indicated by distance in the diagrams. Treatment with $E$. coli 078 altered the composition and structure of the gut microbiota according to PCoA. Treatment with HQD partially inhibited E. coli O78-induced changes in the gut microbiota (Fig. 4).

\subsubsection{HQD regulated structure of the gut microbiota}

The gut microbiota community structure was reported using histograms at the phylum and genus levels. All samples contained abundant Firmicutes, Bacteroidetes and Proteobacteria. Compared with the control group, MG decreased the relative abundance of Bacteroidetes and Firmicutes and increased the proportion of Proteobacteria. These alterations were significantly reversed by HQD administration. Compared with HQD group, the HQD-DZ significantly increased in the abundance of Proteobacteria, which is a marker of intestinal microbial dysregulation, and other herb absent formulae had varying degrees of influence on the three phylum levels. These results indicate that each herb in HQD influences structural segregation of the gut microbiota, in which DZ plays an important role. The ENR group can't recover the changes in the abundance of dominant bacteria caused by $E$. coli 078 . In contrast, the abundance of Proteobacteria was further increased. The results suggest that HQD is more beneficial than ENR in the treatment of E. coli O78 (Fig. 5). 
More than 30 genera were identified in all samples. Bacteroides, Faecalibacterium, Lactobacillus and Prevotella were dominant communities in the control group but were reduced by $E$. coli 078 treatment. Compared with the control group, Escherichia-Shigella becomes the dominant genus, but were reduced by HQD treatment. The results showed that the proportions of most bacteria returned to control levels following HQD treatment.

The gut microbiota community structure of HQD, HQD-GC and HQD-SY were like that of CG group, indicating that HQD, HQD-GC and HQD-SY had protective effect on the structural changes caused by E. coli 078 Challenge. Compared with HQD group, the proportion of Escherichia-Shigella in HQD-DZ and HQD-HQ groups were increased, indicating that DZ and HQ in HQD had a certain regulating effect on the increase EscherichiaShigella (Fig. 6).

\section{Discussion}

Avian pathogenic $E$. coli 078 is the primary cause of colibacillosis, which is associated with remarkable economic losses in the poultry industry worldwide ${ }^{10}$. Antibiotics have been widely used to prevent or combat $E$. coli 078 infection in chicks, but it also has been a surge in antibiotic-resistant bacteria. So, considering the incidence of multi-drug-resistant of $E$. coli 078 and its potential risk for both human and animal health ${ }^{11}$, it is imperative to develop alternative strategies to reduce $E$. coli 078 -infection-induced deleterious effect ${ }^{12}$. Previous studies have shown that HQD has no inhibitory effect on E. coli 078 in vitro, but this effect has been found in vivo studies ${ }^{13}$. Therefore, it is speculated that the treatment effect of HQD on colibacillosis is independent of its direct bactericidal effect, but dependent on the intestinal metabolism, regulation of intestinal microflora structure and immune function.

In the present study, we observed that HQD, by down-regulating the mRNA expression of TLR4, TLR5 and TLR1 5 in the spleen, further decreasing the serum LZM and IL-1 $\beta$, TNF-a, IL-10, IL-6 levels, improves the immune function. Meanwhile, HQD could keep the balance of fecal microbiome of chicks challenged with $E$. coli 078 , which may be function in Toll-like receptors signaling pathway. About herb absent groups, the results shown that SY and DZ play a key role in reducing the level of inflammatory factors and keeping fecal microbiome balance respectively, What's more, we highlighted that HQ is indispensable in HQD, HQ not only play a key role in reducing the level of inflammatory factors, but also keep the balance of fecal microflora.

In agreement with previous study, E. coli 078 challenge led to increasing mortality, decrease average daily gain, and raise organ indexes of heart and liver in chicks ${ }^{14}$, In addition, it also led to severe perihepatitis and pericarditis ${ }^{15}$. In this study, E. coli 078 led to increasing mortality, decreasing average daily gain, and raising organ indexes of heart and liver in chicks, it was attenuated by HQD administration. However, HQD-DZ and HQD-HQ lost this attenuated effect, indicating that HQ and DZ in HQD were indispensable in decreasing mortality and increasing ADG.

In previous study, the method of intramuscular injection was used to challenge the $E$. coli 078 , it allows $E$. coli 078 to crossing the first immune barrier and entering the bloodstream, and then initiates the secretion of inflammatory factors which are critical factors produced by epithelial cells and macrophages following bacterial infections ${ }^{16}$. In the present study, E. coli 078 challenged resulted in increasing LZM, as well as enhancing protein level of inflammatory factors, including IL-1 $\beta$, IL-6, TNF-a and IL-10 in serum of chicks, which 
was attenuated by HQD administration, indicating that HQD could reduce the inflammatory factors level of chicks with colibacillosis. What's more, $\mathrm{HQ}$ and $\mathrm{DZ}$ in HQD play a key role in reducing protein level of $\mathrm{LZM}$, and $\mathrm{HQ}$ and SY play a key role in down-regulating TNF-a.

It's worth noting, in the present study, that $E$. coli 078 challenge led to increasing the level of IL-10 that is an anti-inflammatory factor, has the effect of anti-inflammatory and immunosuppressive. Some researchers believe that anti-inflammatory cytokine IL-10 can reduce the intensity of inflammatory response in severe infection, but on the other hand, the inhibition of the body's defense ability leads to the persistence of microbial infection ${ }^{17}$. Therefore, it is believed that the increase of IL-10 in infection may be a manifestation of severe injury of the body ${ }^{18}$. In present study, E. coli 078 increased protein level of IL-10, it may indicate that E. coli 078 led to severe injury of body.

TLR could identify the microbe related molecular patterns, activate MyD88-dependent signaling pathway, activate transcription factor NF-KB ${ }^{19}$, and produce IL-1 $\beta$, IL-6, TNF- $\alpha$ and other cytokines ${ }^{20}$, enhance the immune function of the body ${ }^{21}$. We observed that E. coli 078 infection led to enhancing the mRNA level of Tolllike receptors in spleen, including TLR4, TLR5 and TLR15, but has no effect in bursal. These up-regulating Tolllike receptors in spleen were accompanied by increasing in serum inflammatory factors, including IL-1 $\beta$, IL-6, TNF- $a$ and IL-10. Therefore, it is suggested that HQD could down-regulate the expressions of TLR4, TLR5 and TLR15 in spleen, and reduce the levels of inflammatory factors, to improve the immune function of infected chicks. Compared with HQD, there was no difference in all groups of herbs absent, indicating that HQD plays a role as a whole in regulating toll-like receptors in spleen.

Intestinal microflora plays an important role in the body's health ${ }^{22}$, which is closely related to the body's immunity, metabolism, digestion and absorption, diseases ${ }^{23}$. Previous studies have reported that Firmicutes can metabolize and produce butyric acid in the intestinal tract of the body ${ }^{24}$, which provides energy for the growth and development of the intestinal cells in the back end and participates in the fermentation of carbohydrates and polysaccharides to improve the transformation of nutrients and energy in the animal body ${ }^{25}$. Enrofloxacin has a good therapeutic effect, but it leads to dysregulation of intestinal flora, which is related to its extensive bactericidal action. It leads to the dissolution of a large number of bacteria in the gut releases more LPS, triggering an inflammatory response, which also explains the increased levels of inflammatory cytokines ${ }^{26}$. Therefore, it is of great significance to maintain the structural stability and diversity of intestinal flora. In the present study, the abundance and diversity of intestinal microflora in chicks infected with $E$. coli 078 were significantly decreased $(p<0.05)$, indicating that $E$. coli 078 broke the immune barrier in vivo following the blood circulation, or $E$. coli 078 challenged the immune function of the body, and increased large number of opportunistic pathogens in the intestine. Finally, E. coli 078 caused the decrease of intestinal microflora diversity and disrupted the balance of intestinal microflora. Fortunately, HQD can effectively attenuate this phenomenon. What's more, compared with HQD, the Shannon index in HQD-DZ group was significantly decreased, indicating that DZ had an important role in maintaining the intestinal microflora structure of chicks challenged with E. coli 078 .

The analysis of intestinal microflora structure in each group showed that the phylum level microflora structure of the model group was changed, the proportion of Proteobacteria increased, and the proportion of Firmicutes decreased. Proteobacteria is considered to be a marker of intestinal microbiota imbalance, and a large number 
of Proteobacteria in the gut reflects stunted growth or an unstable intestinal microbiota structure. But HQD attenuates this change and keeps the intestinal microflora in balance. Compared with HQD, HQD-DZ increased the harmful bacteria Proteobacteria levels, which indicated that DZ in HQD play a key role in keeping fecal microbiome balance in the phylum levels.

Compared with the control group, the genus structure of the microflora in model group was changed, the proportion of Proteobacteria Faecalibacterium, Lactobacillus and Prevotella decreased, and EscherichiaShigella became the dominant genus, as was reversed by HQD treatment similar to control group, indicating that HQD had protective effect on the microflora structural suffering E. coli O78 Challenge. What is more, HQDDZ and HQD-HQ increased the harmful bacteria Escherichia-Shigella, indicating that $\mathrm{HQ}$ and DZ play a key role in keeping fecal microbiome balance in the genus levels in HQD.

\section{Conclusions}

In conclusion, we confirmed HQD by down-regulating the mRNA expression of TLR4, TLR5 and TLR15 in the spleen, further decreasing the serum LZM and IL-1 $\beta$, TNF-a, IL-10, IL-6 levels, improves the immune function, and reverse the change of fecal microbiome in chicks challenged with E. coli O78. About herb absent groups, the results show that SY and DZ play a key role in reducing the level of inflammatory factors and keeping fecal microbiome balance respectively, what's more, we highlighted that $\mathrm{HQ}$ is indispensable in HQD, HQ not only play a key role in reducing the level of inflammatory factors, but also keep the balance of fecal microflora.

\section{Materials And Methods}

\subsection{E. coli 078 preparation and infection}

The E. coli 078 strain (preservation number: CVCC1490; China Veterinary Microorganism Strains Preservation Management Center, Beijing, China) was aerobically cultured in Luria-bacterial liquid medium at $37^{\circ} \mathrm{C}$ for $24 \mathrm{~h}$. The bacterium solution was injected into the chicks for strain rejuvenation. $E$. coli 078 was isolated by coating the liver on MacConkey agar plate at $37^{\circ} \mathrm{C}$ for $24 \mathrm{~h}$. The colonies were suspended in $50 \mathrm{~mL}$ of sterile sodium chloride at $\mathrm{pH}$ 7.0. The number of bacteria was determined by plating dilutions of the suspension onto agar plate. Chicks were intraperitoneally injected with $0.5 \mathrm{~mL}$ of inoculum with a concentration $0.6 \times 10^{9} \mathrm{cfu} / \mathrm{mL}$ at 27 days of age.

\subsection{Preparation of HQD granules for chicks}

According to the prescription of HQD, HQ, SY, DZ and GC were soaked in a ratio of 3:2:2:2 for 60 minutes and boiled for 40 minutes. After filtration, the residue of the $\mathrm{HQ}$ was dried in a drying oven, then the grinded residue was mixed with the concentrated filtrate in the pelletizer, and then cut to wet granules following desiccation. SY, $D Z, G C, H Q$ were not used in the preparation of the granules of HQD-SY, HQD-DZ, HQD-GC, HQD-HQ by the way like the HQD granules. The residue other than HQ was used to prepare HQD-HQ.

\subsection{Chicks and treatments}


A total of 80 1-day-old male Esa brown chicks were randomly assigned into one of 8 treatment groups with 10 chicks per group. Chicks in control group (CG) and model group (MG) were fed a basal diet formulated (Shiyang Agricultural Group Co., LTD, Jinzhong, China) to meet nutritional requirements of chicks during the whole experimental period. Birds in Enrofloxacin Group (ENR) were fed a basal diet supplemented with Enrofloxacin $10 \mathrm{mg} / \mathrm{kg} \cdot \mathrm{BW}$; birds in HQD group (HQD) were fed a basal diet supplemented with HQD granules $500 \mathrm{mg} / \mathrm{kg} \cdot \mathrm{BW}$; birds in group of HQD-HQ were fed a basal diet supplemented with HQD-HQ granules 500 $\mathrm{mg} / \mathrm{kg} \cdot \mathrm{BW}$; birds in group of HQD-SY were fed a basal diet supplemented with HQD-SY granules 500 $\mathrm{mg} / \mathrm{kg} \cdot \mathrm{BW}$; birds in group of HQD-DZ were fed a basal diet supplemented with HQD-DZ granules 500 $\mathrm{mg} / \mathrm{kg} \cdot \mathrm{BW}$; birds in group of HQD-GC were fed a basal diet supplemented with HQD-GC granules 500 $\mathrm{mg} / \mathrm{kg}$.BW. All supplement drugs were administered after intramuscular injection of E. coli 078 at 27 days of age, and they were added up to 32 days of age. All the chicks had free access to food and drinking water during the whole experimental period. The experimental animal protocol for this study was approved by the Animal Care and Use Committee of China Agricultural University.

\subsection{Visceral index}

At 32 days of age, to calculate the chicks' visceral index, the living weight of chicks were weighed and liver, heart, spleen and bursal were collected. The formula is: Visceral index $(\mathrm{g} / \mathrm{kg})=$ visceral weight $(\mathrm{g}) / \mathrm{live}$ weight $(\mathrm{kg})$.

\subsection{Enzyme-linked immunosorbent assay (ELISA)}

To evaluate the effects of drugs on innate immune substances and cytokines in chicks with colibacillosis, the blood was collected, and the serum was separated. The level of LZM (lysozyme) in serum was quantified using ELISA kits for chicken following manufacturer's instructions. The levels of TNF-a, IL-1 $\beta$, IL-6, and IL-10 in serum were quantified using ELISA kits (Biosciences, China) following manufacturer's instructions. Take three samples from each group and repeat each sample three times. The results were expressed as content of LZM or cytokines in per $\mathrm{mL}$ in serum of chicks.

\subsection{Quantitative real-time PCR (RT-qPCR)}

To evaluate the effect of drugs on Toll-like receptor expression in immune organs of chicks with colibacillosis, the bursal and spleen was collected. Total RNA was extracted from spleen and bursal using Trizol reagent (Takara, Japan). According to the Bio-Rad kit, $1 \mu \mathrm{L}$ DNA was taken for polymerase chain reaction. GADPH was used to normalize based on CT value. All primers were synthesized by Wuhan ServiceBio Technology Co., Ltd. Take three samples from each group and repeat each sample three times. Primer sequences are shown in the Table 5 .

Table 5

Primer sequences 


\begin{tabular}{|ll|}
\hline Primer & Primer sequences (5'-3') \\
\hline Gapdh-F & CTGGGGCTCATCTGAAGGGT \\
\hline Gapdh-R & GGACGCTGGGATGATGTTCT \\
\hline TLR4-F & CCAACCCAACCACAGTAGCAT \\
\hline TLR4-R & ATCACCCATTCTTGGTCTTTGC \\
\hline TLR5-F & TCTGACATACGATGACTGCGAT \\
\hline TLR5-R & TGACATCAGAAACATCAGAAGGGT \\
\hline TLR15-F & CGACAACGCCATCACTACCATA \\
\hline TLR15-R & GTTAGCACCAGAACGACAAGGA \\
\hline
\end{tabular}

\subsection{Fecal microflora analysis}

\subsubsection{Extraction of fecal DNA}

At the end of the experiment, the intestinal flora of all groups was studied. The feces of each group of animals were collected and placed into a sterilized cryopreservation tube, which was immediately frozen with liquid nitrogen and stored at $-80^{\circ} \mathrm{C}$ for use. Total genomic DNA from Stool was extracted and purified using DNeasy Power Soil kit (Qiagen, Germany). The integrity and quantity of DNA were determined by agarose gel electrophoresis and spectrophotometry. $500 \mathrm{ng}$ purified DNA was extracted from each sample for subsequent polymerase chain reaction (PCR) and 16S rDNA sequencing.

\subsubsection{S rDNA sequencing}

Appropriate samples were taken and diluted to $10 \mathrm{ng} / \mu \mathrm{L}$ with sterile water. Using the diluted genomic DNA as a template, V3+V4 hypervariable sequences of $16 \mathrm{~S}$ rDNA were amplified by PCR with Takara Ex Taq (Takara, Japan) with barcode-modified universal primers (forward: 343F, 5'-TACGGRAGGCAGCAG-3'; reverse: 798R, 5'AGGGTATCTAATCC T-3'). PCR mixtures contained $15 \mu \mathrm{L}$ of $2 \times$ Gflex PCR Buffer, $2 \mu \mathrm{L}$ of primer mix $\nabla 5 \mathrm{pmol} /$ $\mu \mathrm{L} \otimes \square 5 \mathrm{~L}$ of diluted DNA template $\mathbb{1 0} \mathrm{ng} / \mu \mathrm{L} \otimes \mathbb{\mathrm { N }} 0.6 \mu \mathrm{L}$ of Tks Gflex DNA Polymerase $(1.25 \mathrm{U} / \mu \mathrm{L})$ in a total reaction volume of $30 \mu \mathrm{L}$. PCR conditions were as follows: initial denaturation at $94^{\circ} \mathrm{C}$ for $5 \mathrm{~min}, 26$ cycles of denaturation at $94^{\circ} \mathrm{C}$ for $30 \mathrm{~s}$, annealing at $56^{\circ} \mathrm{C}$ for $30 \mathrm{~s}$, and elongation at $72{ }^{\circ} \mathrm{C}$ for $20 \mathrm{~s}$, with a final elongation step at $72{ }^{\circ} \mathrm{C}$ for $5 \mathrm{~min}$. Amplified products were separated on $2 \%$ agarose gels, the purified product was used as a second PCR template for amplification. The above steps were repeated for purification, and then Qubit dsDNA Assay Kit (Life Technologies, USA) was used for quantitative detection. According to the concentration of PCR products, equal amounts of samples were mixed, Barcoded V3+V4 amplicons were sequenced by the paired-end method with Illumina MiSeq at Shanghai OE Biotech. Co., Ltd. 


\subsubsection{Bioinformatics analysis}

Raw sequences were denoised using Trimmomatic and FLASH software and filtered according to their barcodes and primer sequences with QIIME v.1.5.0. Chimeras were identified and excluded using the UCHIME algorithm v.4.2.40. Optimized, high-quality sequences were clustered into operational taxonomic units (OTUs) at $97 \%$ sequence identity against a subset of the Silva 16 S sequence database (Release 119 1). Taxondependent analysis was carried out using the Ribosomal Database Project (RDP) naive Bayesian classifier, with an $80 \%$ bootstrap cutoff. Alpha diversity (Shannon and Simpson indices), abundance (Chao1 and ACE indices), and Goods coverage and rarefaction were analyzed with mothur v.1.31.2. Principle coordinates analysis (PCoA) was conducted to visualize differences in nasal mucosa community composition. PCoA plots were generated based on Bray-Curtis indices. The linear discriminant analysis effect size (LEfSe) algorithm was used to identify the taxa responsible for the differences between the treatment and control groups. The biomarkers used in the present study had an effect-size threshold of two.

\subsection{Statistical analysis}

Statistical analysis of data from ELISA and RT-qPCR assays was carried out using a one-way analysis of variance (ANOVA) was performed to detect differences between mean values, which were then analyzed further for significance with LSD test. The chi-square test was used to analyze the differences in mortality. In all cases, $p<0.05$ were considered significant.

\section{Declarations}

\section{Acknowledgements}

Thanks to Professor Xichun Zhang providing language help and proofing the article. Thanks to Professor Jianxin Zhang providing animal lab.

\section{Funding}

This research was funded by Basic Research project of Shanxi Province[grant number 20210302123233]; Science Research Start-up Fund for Doctor of Shanxi Province [grant number 2020BKS01]; Science Research Start-up Fund for Doctor of Shanxi University of Chinese Medicine [grant number 20170101]; and the Key Research and Development Program of Shanxi Province [grant number 201903D221013].

\section{Author information}

\section{Affiliations}

College of Veterinary Medicine, Shanxi Agricultural University, Taigu, PR China 
Junyan Wang, Jianjian Feng, Linjie Bao, Yihe Wu \&ShumingChen

College of Pharmaceutical \& Food Engineering, Shanxi University of Chinese Medicine, Yuci, PR China

Rui Li, Yong Liu, Chensheng Gu, Haili Wang \& Xichun Zhang

\section{Author contributions}

Yong Liu: Conceptualization, Methodology, Software. Junyan Wang, Rui Li: Data curation, Writing- Original draft preparation. Yihe Wu, Jianjian Feng: Visualization, Investigation. Shuming Chen, Xichun Zhang: Supervision. Linjie Bao, Chensheng Gu: Software, Validation. Haili Wang, Junyan Wang: Writing- Reviewing and Editing.All authors read and approved the final manuscript.

\section{Corresponding authors}

Correspondence to Xichun Zhang or Shuming Chen.

\section{Ethics declarations}

\section{Ethics approval and consent to participate}

The experimental animal protocol for this study was approved by the Animal Care and Use Committee of China Agricultural University.

\section{Competing interests}

The authors declare no competing financial interests.

\section{Guidelines and Regulations}

This study is reported in accordance with ARRIVE guidelines.

\section{References}

1. Chen, G.;et al., 2016. Protective effects of Huangqin Decoction against ulcerative colitis and associated cancer in mice. Oncotarget 7(38): 61643-61655.

2. Carli, S. D.; et al., 2015. Virulence gene content in Escherichia coli isolates from poultry flocks with clinical signs of colibacillosis in Brazil. Poultry Science 94(11): 2635-2640.

3. Obeng, A. S.;et al., 2012. Antibiotic resistance, phylogenetic grouping and virulence potential of Escherichia coli isolated from the faeces of intensively farmed and free range poultry. Vet Microbiol 154(3-4): 305315. 
4. Ying, Z.;et al., 2015. Huangqin-Tang ameliorates TNBS-induced colitis by regulating effector and regulatory CD4+ T cells. Biomed Res Int 2015: 102021.

5. Yao;et al., 2019. Anti-Inflammatory effects of Huangqin Decoction on dextran sulfate sodium-induced ulcerative colitis in mice through regulation of the gut microbiota and suppression of the Ras-PI3K-Akt-HIF1a and NF-KB pathways. Frontiers in pharmacology 10: 1552-1552.

6. Wu, N.;et al., 2021. Effect of Huangqin Decoction on pyroptosis pathway of NLRP3/caspase-1 in mice with ulcerative colitis. China journal of Chinese materia medica 46(5): 1191-1196.

7. Wang, K.;et al., 2020. Mechanism of Jiawei Huangqin decoction for treating ulcerative colitis in mice: the role of STAT3/NF-kB/IL-6 pathway. Nan Fang Yi Ke Da Xue Xue Bao 40(2): 196-202.

8. Yang, Y.;et al., 2017. Gut microbiota drives the attenuation of dextran sulphate sodium-induced colitis by Huangqin Decoction. Oncotarget 8(30): 48863-48874.

9. Muegge, B. D.;et al., 2011. Diet drives convergence in gut microbiome functions across mammalian phylogeny and within humans. Science 332(6032): 970.

10. Xing, Z.;et al., 2020. Disequilibrium in chicken gut microflora with avian colibacillosis is related to microenvironment damaged by antibiotics science direct. Science of The Total Environment.

11. Subedi, M.;et al., 2018. Antibiotic resistance pattern and virulence genes content in avian pathogenic Escherichia coli (APEC) from broiler chickens in Chitwan, Nepal. BMC Veterinary Research 14(1): 113.

12. Johar, A.;et al., 2021. Antibiotic resistance and virulence gene patterns associated with avian pathogenic Escherichia coli (APEC) from broiler chickens in qatar. Antibiotics 10(5): 564.

13. Ma, Q.;et al., 2018. San Wu Huangqin Decoction, a Chinese herbal formula, inhibits influenza a/PR/8/34 (H1N1) virus infection in vitro and in vivo. Viruses 10(3): 117.

14. Huang, L.;et al., 2018. Effects of the dietary probiotic, enterococcus faecium NCIMB11181, on the intestinal barrier and system immune status in Escherichia coli 078-challenged broiler chickens. Probiotics Antimicrobial Proteins 1-11.

15. Wernicki, A.;et al., 2017. Bacteriophage therapy to combat bacterial infections in poultry. Virology journal 14(1): 179.

16. Zhong, X.;et al., 2014. Polyphenol extracts from punica granatum and terminalia chebula are antiinflammatory and increase the survival rate of chickens challenged with Escherichia coli. Biological pharmaceutical bulletin 37(10): 1575-1582.

17. Houra, M.;et al., 2020. The roles played by IL-10, IL-23 and IL-17A in term delivery. Journal of NeonatalPerinatal Medicine 14(1): 1-9.

18. Goodman, A. L.;et al., 2011. Extensive personal human gut microbiota culture collections characterized and manipulated in gnotobiotic mice. Proceedings of the National Academy of Sciences 108(15): 62526257.

19. Luo, H.,et al., 2012. Role of TLR4/NF-KB in damage to intestinal mucosa barrier function and bacterial translocation in rats exposed to hypoxia. Plos One 7(10): 46291.

20. Wullaert, A. 2010. Role of NF-кB activation in intestinal immune homeostasis. International Journal of Medical Microbiology 300(1): 49-56. 
21. Song, D. H. and J. O. Lee 2012. Sensing of microbial molecular patterns by Toll-like receptors. Immunol Rev 250(1): 216-229.

22. Grond;et al., 2018. The avian gut microbiota: community, physiology and function in wild birds. Journal of Avian Biology 49.

23. Cox, L.;et al., 2014. Altering the intestinal microbiota during a critical developmental window has lasting metabolic consequences. Cell 158(4): 705-721.

24. Peng, L.;et al., 2021. Protective effects of gut microbiota and gut microbiota-derived acetate on chicken colibacillosis induced by avian pathogenic Escherichia coli. Veterinary microbiology 261: 109187.

25. Jumpertz, R.;et al., 2018. Energy-balance studies reveal associations between gut microbes, caloric load, and nutrient absorption in humans. The American journal of clinical nutrition 94 (1): 58-65.

26. Temmerman, R.;et al., 2021. Enrofloxacin dose optimization for the treatment of Colibacillosis in broiler chickens using a drinking behaviour pharmacokinetic model. Antibiotics 10(5).

\section{Figures}

A
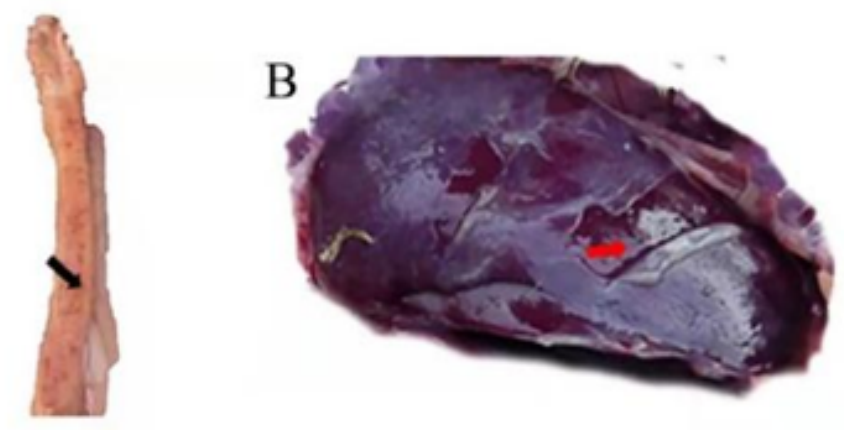

C

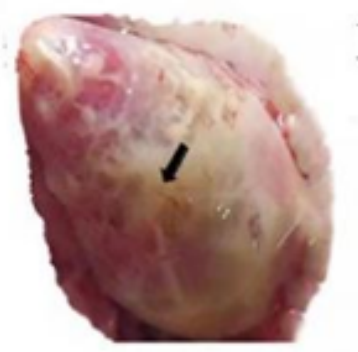

D

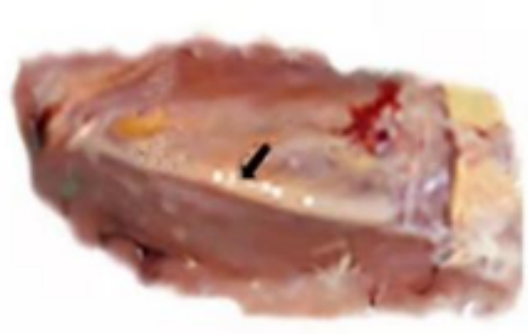

\section{Figure 1}

Colibacillosis chicks show severe intestinal

bleeding site $(A)$, perihepatitis $(B)$, pericarditis $(C)$ and

granuloma (D). 

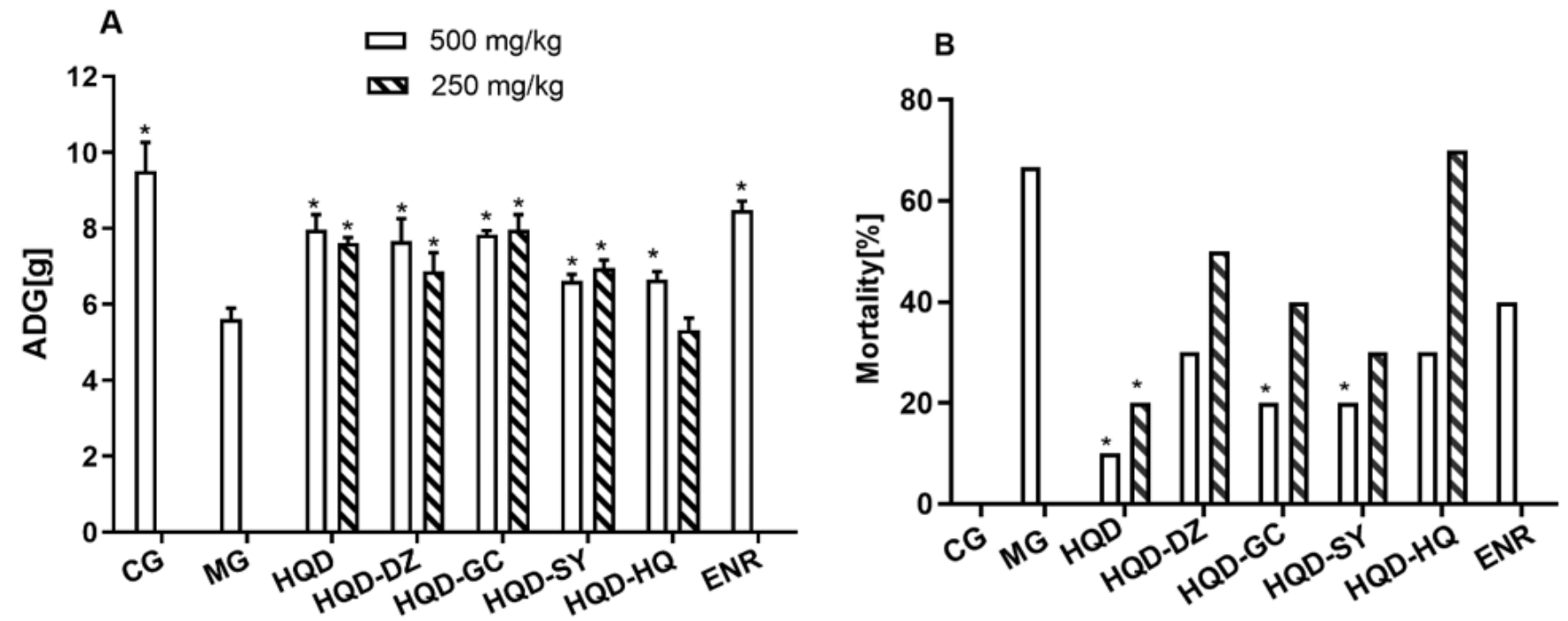

Figure 2

Average daily gain (A) and mortality (B) of chicks in Control group (CG), Model group (MG), Huangqin Decoction group (HQD); and that in HQD group of DZ absent (HQD-DZ), GC absent (HQD-GC), SY absent (HQDSY), HQ absent (HQD-HQ); and in Enrofloxacin group (ENR). * Statistically significant $p<0.05$ versus model.

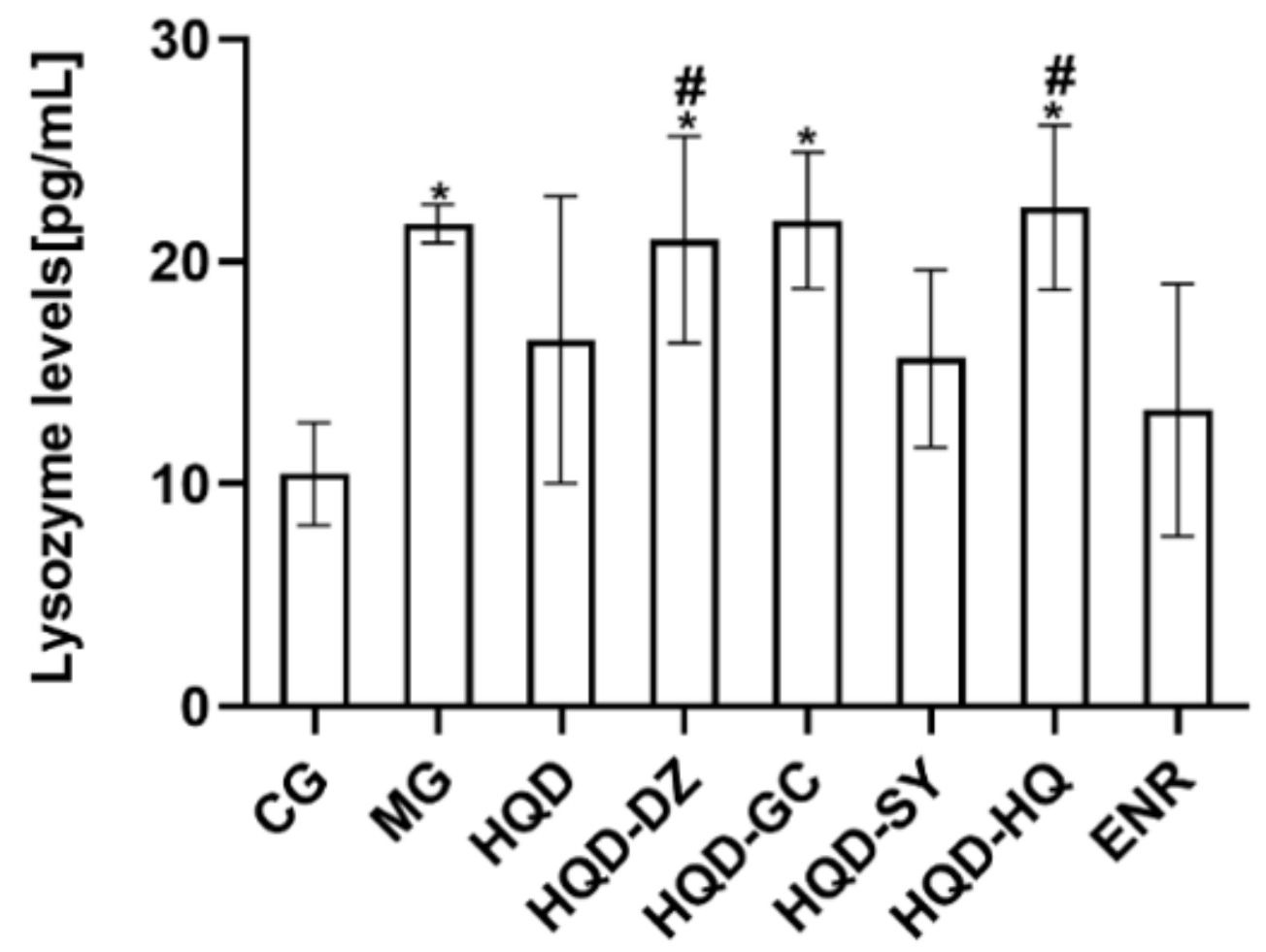


Figure 3

Lysozyme levels of chicks in Control group (CG), Model group (MG), Huangqin Decoction group (HQD); and that in HQD group of DZ absent (HQD-DZ), GC absent (HQD-GC), SY absent (HQD-SY), HQ absent $(\mathrm{HQD}-\mathrm{HQ})$; and in Enrofloxacin group (ENR). Data are expressed as mean \pm SEM. Statistical significance: * $p<$ 0.05 versus model, $\# p<0.05$ versus HQD.

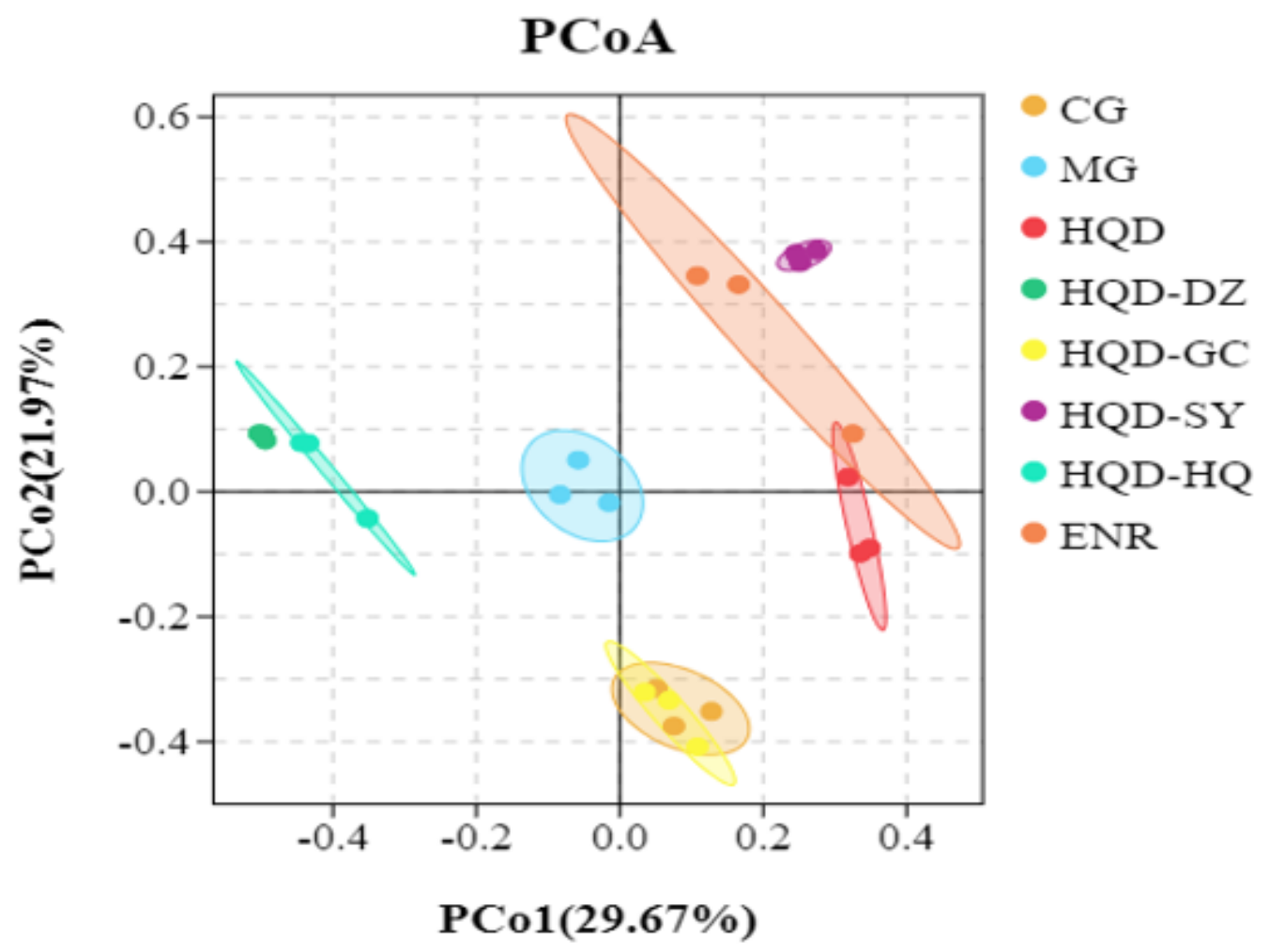

Figure 4

Analysis of intestinal microorganism of chicks in Control group (CG), Model group (MG), Huangqin Decoction group (HQD); and that in HQD group of DZ absent (HQD-DZ), GC absent (HQD-GC), SY absent (HQD-SY), HQ absent (HQD-HQ); and in Enrofloxacin group (ENR). 


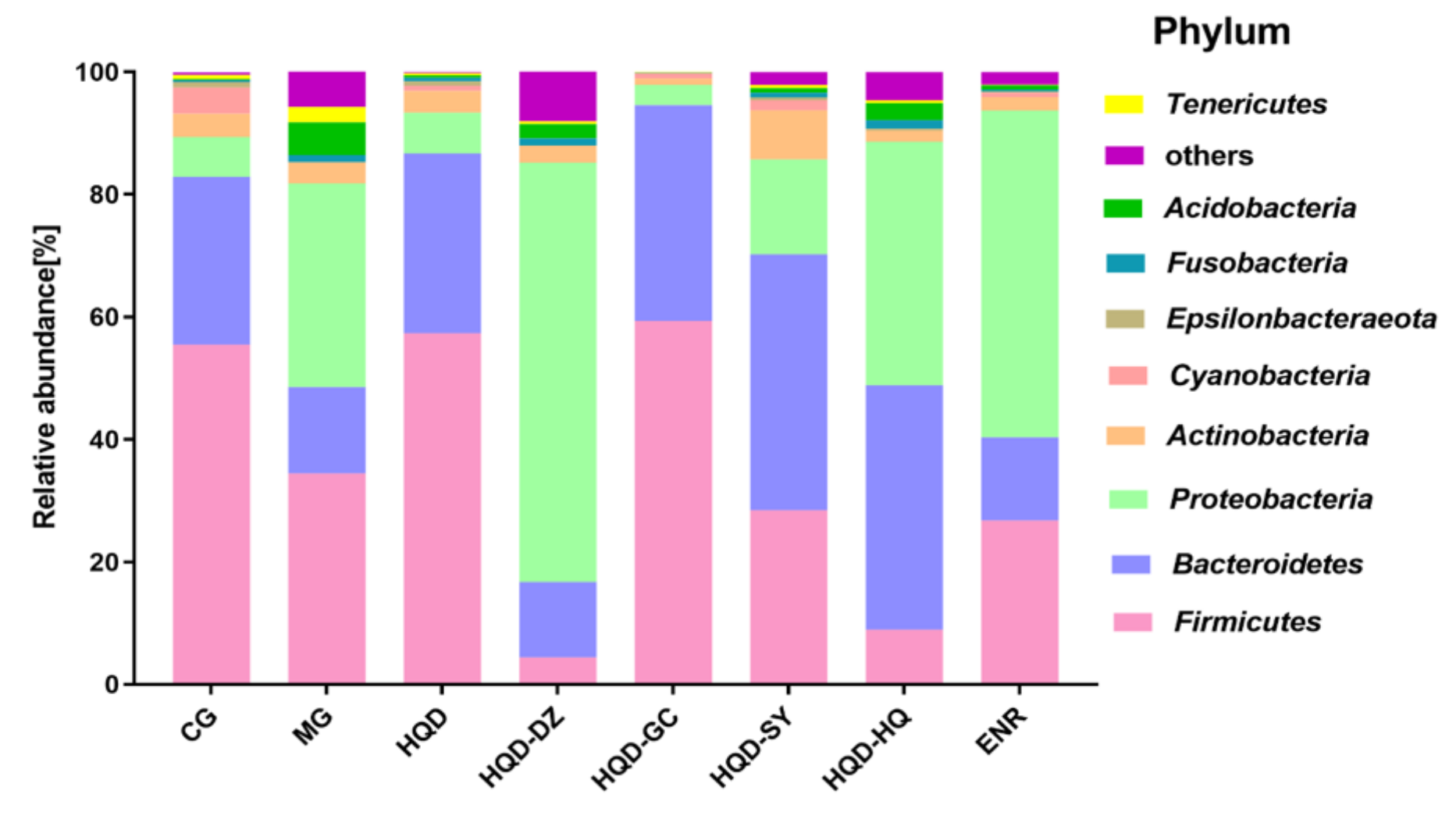

Figure 5

Phylum level analysis of intestinal microorganism relative abundance of chicks in Control group (CG), Model group (MG), Huangqin Decoction group (HQD); and that in HQD group of DZ absent (HQD-DZ), GC absent (HQD-GC), SY absent (HQD-SY), HQ absent (HQD-HQ); and in Enrofloxacin group (ENR), (each color represents one bacterial phylum). 


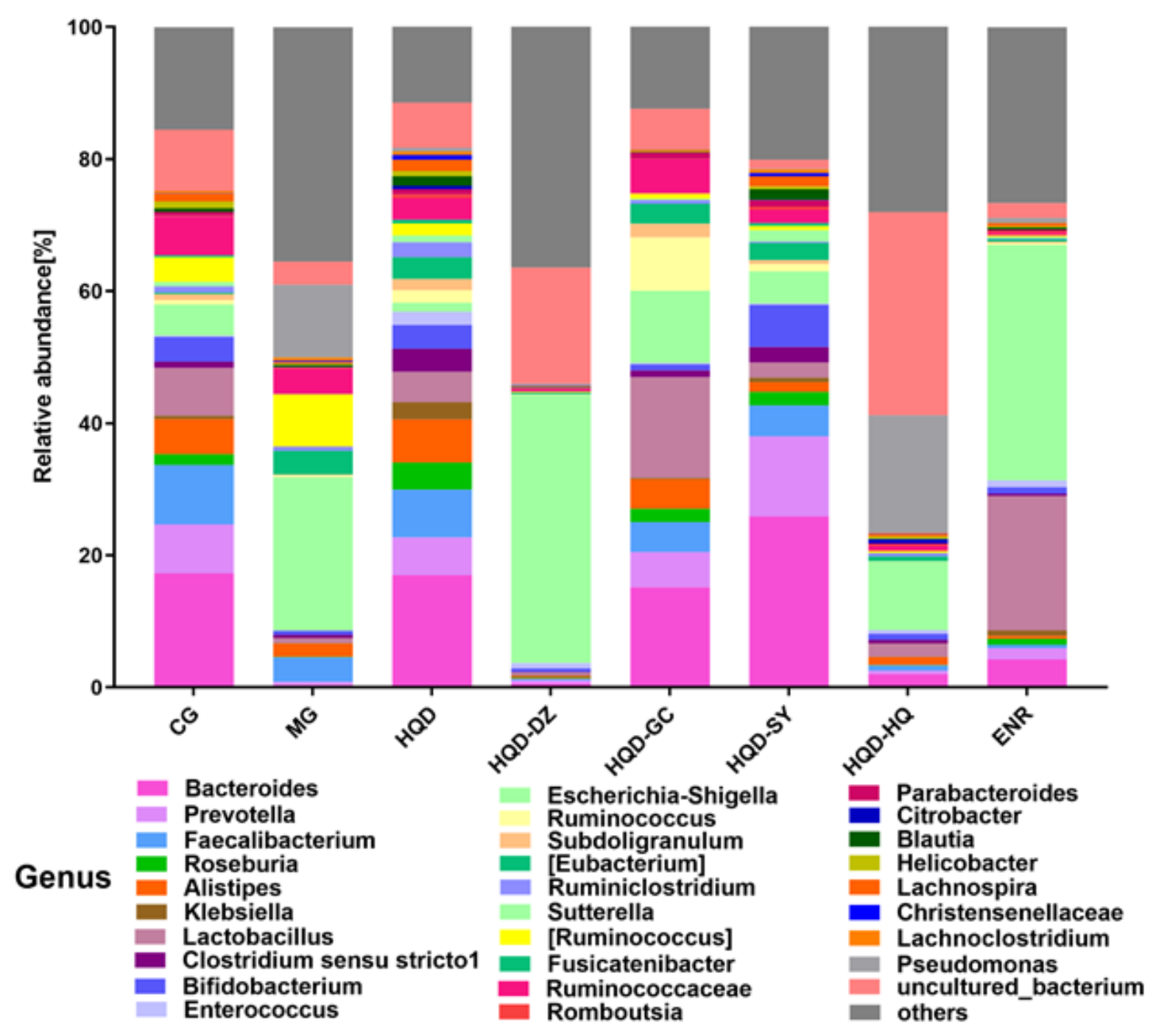

Figure 6

Genus level analysis of intestinal microorganism relative abundance of chicks in Control group (CG), Model group (MG), Huangqin Decoction group (HQD); and that in HQD group of DZ absent (HQD-DZ), GC absent (HQD-GC), SY absent (HQD-SY), HQ absent (HQD-HQ); and in Enrofloxacin group (ENR), (each color represents one bacterial genus).

\section{Supplementary Files}

This is a list of supplementary files associated with this preprint. Click to download.

- GraphicalAbstarct.png 